\title{
AN APPROACH FOR TRACKING ANALYSIS FOR LINEAR SYSTEMS WITH DEAD-ZONE NONLINEARITY APPLIED TO A HYDRAULIC ACTUATOR
}

\author{
VANessa Dilda* EugêNio B. CASTElan* \\ ${ }^{*}$ Control of Mechatronic Systems Team, \\ DAS/CTC/UFSC, 88040-900 \\ Florianópolis, SC, Brazil
}

Emails: dilda@das.ufsc.br, eugenio@das.ufsc.br

\begin{abstract}
Conditions for tracking analysis for linear systems with dead-zone nonlinearity are proposed by using LMI (Linear Matrix Inequalities) based algorithms. We treat the nonlinearity as a bounded disturbance and use the ultimate boundedness stability concept to estimate a region where the tracking error converges in a finite time and remains inside. The dead-zone nonlinearity is a common imperfection of directional control valves, which is a source of performance and stability degradations in control applications. In order to verify the obtained theoretical results using an real system, we consider a 3rd-order linear model of a hydraulic actuator system.
\end{abstract}

Keywords- Ultimate Boundedness Stability, Dead-zone, Hydraulic Actuator Systems, Tracking Analysis, Linear Matrix Inequalities.

\section{INTRODUCTION}

Real control systems are generally subject to the existence of nonlinearities in the control loop, such as saturation, dead-zone, hysteresis, backlash and others (Khalil, 2002; Vidyasagar, 1993; Hu and Lin, 2001; Tarbouriech, Garcia, Gomes da Silva Jr and Queinnec, 2011). The presence of these nonlinearities is induced by the physical limitations of the actuators or for security reasons and generates generally a degradation of performances, stability and tracking properties. A very common nonlinearity in physical systems, as in the case of hydraulic valves (Merritt, 1967), is the dead-zone.

If we do not consider the presence of input references, a main difficulty for treating the stability analysis and the synthesis of stabilizing control laws involving systems subject to dead-zone nonlinearity is as follows: when the control signal is small enough, the output of the dead-zone is zero and, therefore, the control system behaves in open-loop in a neighborhood of the origin. Thus, the asymptotic stability of the origin of a linear system with actuators subject to deadzone nonlinearities is possible only for an asymptotically stable open-loop system. In other cases, the dead-zone causes a loss of local stability, implying that the trajectories of the closed-loop system cannot be expected to converge to the origin. Equilibrium points distinct from the origin or a limit cycle may appear (Khalil, 2002). Nevertheless it may be possible to define a $C$-set (Blanchini, 1999), in which, for any initial condition, the trajectory enters in a finite time (depending on the initial condition) and does not escape of it. Such a property is called in the literature ultimate boundedness or practical stability.

The stability and the stabilization of systems with dead-zone have generated a rich literature in control systems theory, by considering several approaches. Let us cite a few of them. The compensation scheme based on the inverse of a dead-zone and adaptive control has been early presented in Tao and Kokotović (1994). A natural piecewise affine system approach has been proposed in Fong and Hsu (2000). The characteristic of a unique (local or global) cone bounded sector condition (Tarbouriech et al., 2006; Castelan et al., 2008) can be replaced by a bandboundedones (Hsu and Fong, 2001) to cover more generic nonlinearities or by a generalized sector condition (Turner, 2006; Tarbouriech, Queinnec, Alamo, Fiacchini and Camacho, 2011) related to anti-windup techniques (Tarbouriech et al., 2009) and convex differential inclusions (Alamo et al., 2009).

More recently, in Dilda et al. (2013) LMI sufficient conditions were provided for the ultimate boundedness analysis and syntheses of an interconnection between a linear system and a dead-zone. In this manuscript, it was proposed a quite general and sophisticate sector conditions model for this nonlinearity. In Milhomem (2013) a simpler approach is used to treat the stability and stabilization of systems subject to polytopic bounded disturbances and the author shows how this result can be used for analysis and synthesis of stabilizing control laws in the case of linear systems with actuators subject to dead-zones (see also Pomar et al. (2012)). Thus, this last result is used in the present work to propose an approach for tracking analysis for linear systems with dead-zone nonlinearity and it is applied to a hydraulic actuator linear model.

The dead-zone nonlinearity is a common imperfection of mechanical system components and mainly of closed center valves when the land width is greater than the port width at neutral spool position (Virvalo, 1997), such as in the case of proportional directional valves. The hydraulic actuator system, considered in this paper, is composed of a 4-way proportional directional valve and a hydraulic cylinder double-rod. An advantage in using hydraulic actuators in control systems is their capacity to develop high forces in relation to its size or weight, and also to get fast responses to 
the commands at starts, stops or inversions of speed without damages to the mechanical parts. However, the intrinsic nonlinear characteristics of hydraulic actuators make it hard to control. Thus we consider a linear system of third order that describes the dynamic behavior of the hydraulic actuator to validate the obtained theoretical results.

In the two next sections, we consider a general linear system represented in the state space with actuators subject to a dead-zone nonlinearity, that allows us to state the considered control problem and present the tools for analyzing the effects of the dead-zone nonlinearity on the behaviour of the closed-loop system. Finally, we present the third order model of the hydraulic actuator and we apply and comment the results and same simulations.

Notation: $\mathbb{R}, \mathbb{N}, \mathbb{N}^{*}$ are respectively the sets of real numbers, of natural integers and of strictly positive integers. $A^{\prime}$ denotes the transpose of matrix $A$. $I_{n}$ (resp. $0_{n}$ ) and $0_{m \times n}$ are the $n$-order identity (null) matrix and the $m \times n$-order null matrix, respectively. $\operatorname{Re}(\cdot)$ stands for the real part of a scalar. The symbol $*$ stands for symmetric block in matrices. The ellipsoidal $\mathcal{E}(S)$ associated with $S>0$ is given by $\left\{x \in \mathbb{R}^{n} ; x^{\prime} S x \leq 1\right\}$.

\section{PROBLEM STATEMENT}

Consider a linear time-invariant continuous-time system with actuators subject to dead-zone nonlinearity, defined by:

$$
\begin{aligned}
& \dot{x}(t)=A x(t)+B \mathrm{dz}(u(t)), \\
& y(t)=C x(t),
\end{aligned}
$$

where $x(t) \in \mathbb{R}^{n}, u(t) \in \mathbb{R}^{m}$ and $y(t) \in \mathbb{R}^{p}$ are the state, control input and output vectors, respectively; $A, B$ and $C$ are matrices of appropriate dimensions. The objective of the present work is the analysis of some tracking properties of the output of the closed-loop system under an integral state-feedback control law, when subject to a bounded but possibly varying reference vector $r(t) \in \mathfrak{R}^{p}$. For simplicity of the presentation and considering the example treated later, we consider that system (1)-(2) is monovariable: $m=p=1$.

For this system, the following assumptions are considered:

1. the pair $(A, B)$ is assumed to be controllable and $(A, C)$ is assumed to be observable;

2. the following condition (Young and Willems, 1972), is verified:

$$
\operatorname{rank}\left(\left[\begin{array}{cc}
A & B \\
C & 0
\end{array}\right]\right)=n+1
$$

3. without loss of generality, it is considered that $C=\left[\begin{array}{ll}1 & 0_{1 \times(n-1)}\end{array}\right]$.

The dead-zone is a decentralized and symmetric nonlinearity, defined by:

$$
\mathrm{dz}(u(t))=\left\{\begin{array}{rll}
u(t)-\rho, & \text { if } & u(t)>\rho, \\
0, & \text { if } & |u(t)| \leq \rho, \\
u(t)+\rho, & \text { if } & u(t)<-\rho,
\end{array} .\right.
$$

If we do not consider the presence of input references, when the control signal $u(t)$ is small enough, the output of the dead-zone is zero and, therefore, the control system behaves in open-loop in a certain region around the origin. Thus, the asymptotic stability of the origin of system (1) is only possible for asymptotically stable open-loop systems, that is when $\mathrm{A}$ is Hurwitz. In any other case, the dead-zone prevents the trajectories from reaching the origin, even though they will possibly attain a bounded region around it and remaining inside. Since asymptotic stability cannot be ensured in this case, we look for the ultimate boundedness property (Khalil, 2002) ${ }^{1}$.

A study about the effect of the dead-zone in the system (1), was developed in Dilda et al. (2013). In this manuscript, it was proposed a convenient reformulation of the system (1), by considering the statefeedback control low $u(t)=K x(t)$ and the dual nonlinearity of the dead-zone, the saturation $\operatorname{sat}(u(t))=$ $u(t)-\mathrm{dz}(u(t))$, which allows to use the fact that $(A+B K)$ is Hurwitz. This reformulation is given by:

$$
\dot{x}(t)=(A+B K) x(t)-B \operatorname{sat}(K x(t)) .
$$

In the present work, the interest is to evaluate the effect of the dead-zone nonlinearity in the presence of the reference input, with the tracking error given by $e(t)=y(t)-r(t)$.

More specifically, we consider that a statefeedback control law with integral term is applied:

$$
u(t)=K x(t)+K_{I} \xi(t),
$$

where $\xi(t)=\int_{0}^{T} e(t) d t, K \in \mathbb{R}^{1 \times n}$ and $K_{I} \in \mathbb{R}$. Then $\dot{\xi}(t) \triangleq e(t)=C x(t)-r(t)$. To carry out the analysis of this tracking problem, from Assumption 3 we consider the partition $x(t)=\left(\begin{array}{lll}x^{1}(t) & x^{2}(t)\end{array}\right)^{\prime}$, where $x^{1}(t)=$

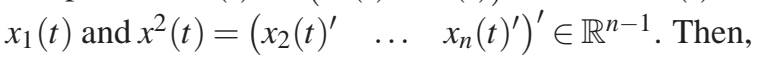
the tracking error can be written as

$$
e(t)=x^{1}(t)-r(t) .
$$

Considering the extended vector $z(t)=$ $\left[\begin{array}{ll}x(t)^{\prime} & \xi(t)^{\prime}\end{array}\right]^{\prime}=\left[\begin{array}{lll}x^{1}(t) & x^{2}(t) & \xi(t)^{\prime}\end{array}\right]^{\prime}$, the closedloop system formed by (1), (2) and (5) can be rewritten as

$$
\begin{aligned}
\dot{z}(t) & =(\mathcal{A}+\mathcal{B} \mathcal{K}) z(t)-\mathcal{B} \operatorname{sat}(u(t))+\mathcal{B}_{r} r(t), \\
u(t) & =\mathcal{K} z(t), \\
y(t) & =\mathcal{C} z(t),
\end{aligned}
$$

where

$$
\begin{gathered}
\mathcal{A}=\left[\begin{array}{cc}
A & 0_{n \times 1} \\
C & 0
\end{array}\right], \mathcal{B}=\left[\begin{array}{l}
B \\
0
\end{array}\right], \mathcal{B}_{r}=\left[\begin{array}{c}
0_{n \times 1} \\
-1
\end{array}\right], \\
\mathcal{C}=\left[\begin{array}{ll}
C & 0
\end{array}\right]=\left[\begin{array}{ll}
1 & 0_{1 \times n}
\end{array}\right], \mathcal{K}=\left[\begin{array}{ll}
K & K_{I}
\end{array}\right],
\end{gathered}
$$

with $\mathcal{A}_{\mathrm{cl}}=(\mathcal{A}+\mathcal{B} \mathcal{K})$ is Hurwitz. 
In the ideal case without dead-zone, the closedloop system is given by

$$
\dot{z}_{\text {id }}(t)=\mathcal{A}_{c l} z_{i d}(t)+\mathcal{B}_{r} r(t)
$$

where

$$
z_{i d}(t)=\left[\begin{array}{lll}
x_{i d}^{1}(t) & x_{i d}^{2}(t) & \xi_{i} d(t)^{\prime}
\end{array}\right]^{\prime}
$$

Thus, we are now interested in evaluating the difference between the tracking error generated by the systems (7) and (10), that is $e_{\Delta}(t) \triangleq e(t)-e_{i d}(t)=$ $\left(x^{1}(t)-r(t)\right)-\left(x_{i d}^{1}(t)-r(t)\right)$. Hence,

$$
e_{\Delta}(t) \triangleq x^{1}(t)-x_{i d}^{1}(t) .
$$

In this way, let us consider the extended difference vector

$$
z_{\Delta}(t)=z(t)-z_{i d}(t)=\left[\begin{array}{c}
x^{1}(t)-x_{i d}^{1}(t) \\
x^{2}(t)-x_{i d}^{2}(t) \\
\xi(t)-\xi_{i d}(t)
\end{array}\right]=\left[\begin{array}{c}
e_{\Delta} \\
z_{\Delta}^{2}
\end{array}\right]
$$

From (7) and (10), we have

$$
\dot{z}_{\Delta}(t)=\mathcal{A}_{c l} z_{\Delta}(t)-\mathcal{B} \operatorname{sat}(u(t)),
$$

where $u(t)$ is defined by (8).

In special, from (13), we can assess the ultimate boundedness behavior of $z_{\Delta}(t)$ and in consequence, of $e_{\Delta}(t)=x^{1}(t)-x_{i d}^{1}(t)$, which corresponds to the first coordinates of $z_{\Delta}(t)$.

Thus, we reformulate the considered tracking problem as follows:

Determine a constant $\beta>0$, as small as possible, then $\bar{t}>0$ such that

$$
\left|e_{\Delta}(t)\right|<\beta, \forall t \geq \bar{t}>0 .
$$

\section{ULTIMATE BOUNDEDNESS ANALYSIS}

Considering that sat $(u(t))$ is limited in amplitude, we consider to rewrite system (13) as follows (Milhomem, 2013; Pomar et al., 2012):

$$
\dot{z}_{\Delta}(t)=\mathcal{A}_{c l} z_{\Delta}(t)-\mathcal{B} w(t),
$$

where $w(t)$ is considered to be a bounded disturbance in a compact polytopic set $W \subset \mathbb{R}^{m}$, containing the origin, given by:

$W=\left\{w(t) \in \mathbb{R}^{n} / w(t)=\sum_{j=1}^{N} \alpha_{j}(t) w_{j}, \sum_{j=1}^{N} \alpha_{j}(t)=1, \alpha_{j}(t) \geq 0\right\}$,

in which $\left\{w_{1}, \ldots, w_{N}\right\} \in \mathbb{R}^{n}$ are vertices of a polytope. In the present case, $W=\left\{w(t) \in \mathbb{R}^{n} / w(t) \subseteq[-\rho, \rho]\right\}$, because of the limits of the saturation function ${ }^{2}$.

In order to prove the ultimate boundedness property of the trajectories of the system (15), we consider a quadratic candidate Lyapunov function, $V\left(z_{\Delta}\right)=$ 
Note that the inequality (17) is a BMI (Bilinear Matrix Inequalities) due to the presence of a product of variables, $\tau \mathcal{P}$. Nevertheless, when $\tau$ is fixed, the inequality becomes an LMI. A line-search on the scalar $\tau$ is possible to reach the solution of Optimization Problem (20).

Remark 1 From (12), we can consider the partitioning

$$
\mathcal{P}=\left[\begin{array}{ccc}
\mathcal{P}_{11} & \mathcal{P}_{12} & \mathcal{P}_{13} \\
* & \mathcal{P}_{22} & \mathcal{P}_{23} \\
* & * & \mathcal{P}_{33}
\end{array}\right]=\left[\begin{array}{cc}
\mathcal{P}_{11} & \overline{\mathcal{P}}_{12} \\
* & \overline{\mathcal{P}}_{22}
\end{array}\right]
$$

with $\mathcal{P}_{11}>0 \in \mathbb{R}, \overline{\mathcal{P}}_{12} \in \mathbb{R}^{1 \times n}$ and $\overline{\mathcal{P}}_{22} \in \mathbb{R}^{n \times n}$. Thus we have

$$
\left[\begin{array}{l}
e_{\Delta} \\
z_{\Delta}^{2}
\end{array}\right]^{\prime}\left[\begin{array}{cc}
\mathcal{P}_{11} & \overline{\mathcal{P}}_{12} \\
* & \overline{\mathcal{P}}_{22}
\end{array}\right]\left[\begin{array}{l}
e_{\Delta} \\
z_{\Delta}^{2}
\end{array}\right] \leq 1
$$

If we consider $z_{\Delta}^{2}=-\overline{\mathcal{P}}_{22}^{-1} \overline{\mathcal{P}}_{12} e_{\Delta}+\bar{z}_{\Delta}^{2}$, we can rewrite (21) as

$$
\left[\begin{array}{l}
e_{\Delta} \\
\bar{z}_{\Delta}^{2}
\end{array}\right]^{\prime}\left[\begin{array}{cc}
\mathcal{P}_{11}-\overline{\mathcal{P}}_{12} \overline{\mathcal{P}}_{22}^{-1} \overline{\mathcal{P}}_{12}^{\prime} & 0 \\
* & \overline{\mathcal{P}}_{22}
\end{array}\right]\left[\begin{array}{l}
e_{\Delta} \\
\bar{z}_{\Delta}^{2}
\end{array}\right] \leq 1,
$$

Hence, by considering that $\mathcal{P}$ is a solution of the Optimization Problem (20), the associated solution (14) of the considered analysis problem is:

$$
\left|e_{\Delta}\right|<\beta=\frac{1}{}
$$


Table 1: Parameters of the hydraulic actuator. 


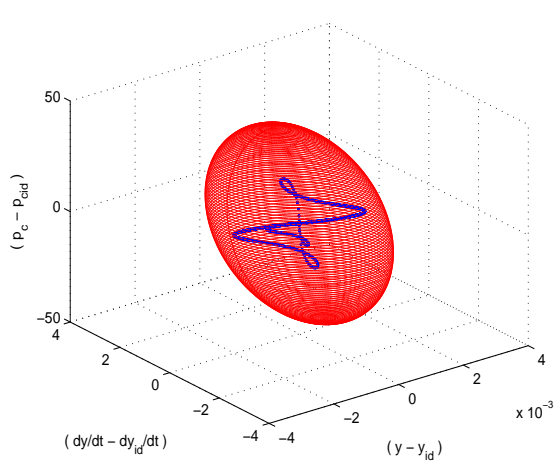

Figure 5: Projection of the UB region and the trajectories.

The Figure 6 shows the limits and time evolution of the tracking error difference.

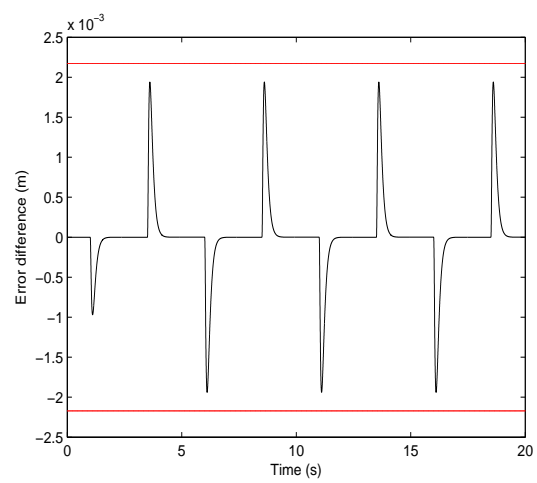

Figure 6: Limits and time evolution of the tracking error difference, $e_{\Delta}$.

\section{CONCLUSION}

We have presented an approach for tracking analysis for linear systems with dead-zone nonlinearity applied to a hydraulic actuator. The nonlinearity has been considered as a bounded disturbance, and the conditions for tracking analysis have been formulated using an LMI based optimization algorithm. It is possible to conclude that for any reference bounded signals, the tracking error will stay confined in a ultimate bounded region. This initial analysis result of the stability analysis can be extended to formulate conditions for synthesis and design of a state-feedback with integral term control that minimizes the effects of dead-zones on the closed-loop system.

\section{Agradecimentos}

The authors thanks CAPES and CNPq for the financial support.

\section{References}

Alamo, T., Cepeda, A., Fiacchini, M. and Camacho, E. (2009). Convex invariant sets for discrete-time Lur'e systems, Automatica 45(4): 1066-1071. DOI: 10.1016/j.automatica.2008.11.013
Blanchini, F. (1999). Set invariance in control, Automatica 35: 1747-1767. DOI: 10.1016/S0005-1098(99)00113-2

Castelan, E., Tarbouriech, S. and Queinnec, I. (2008). Control design for a class of nonlinear continuous-time systems, Auto-matica 44(8): 2034-2039. DOI: 10.1016/j.automatica.2007.11.013

De Negri, V. and Kinceler, R. (2001). A new flow force compensated control valve conception and mathematical modelling, Proceedings of the 16th Brazilian Congress of Mechanical Engineering, Vol. 13, pp. 219-228.

Dilda, V., Jungers, M. and Castelan, E. (2013). Uniformly Ultimate Boundedness Analysis and Synthesis for Linear Systems with Dead-zone in the Actuator, Submitted for publication in the International Journal of Robust and Nonlinear Control .

Fong, I. and Hsu, C. (2000). State feedback stabilization of single input systems through actuators with saturation and deadzone characteristics, Proceedings of the 39th IEEE Conference on Decision and Control, Sydney, Australia, Vol. 4, pp. 32663271.

Hsu, C. and Fong, I. (2001). Ultimate boundedness control of linear systems with band-bounded nonlinear actuators and additive measurement noise, Systems \& Control Letters 43(5): 329336. DOI: 10.1016/S0167-6911(01)00115-3

Hu, T. and Lin, Z. (2001). Control systems with actuator saturation. Analysis and design, Birkhäuser. DOI: 10.1007/978-1-4612-0205-9

Khalil, H. (2002). Nonlinear systems, Vol. 3, Prentice Hall.

Merritt, H. E. (1967). Hydraulic control systems, Wiley New York.

Milhomem, R. (2013). Análise e síntese de sistemas sujeitos a uma classe de perturbações limitadas e aplicações, Master's thesis, Florianópolis, Brasil: Universidade Federal de Santa Catarina.

Muraro, I. (2009). Estudo das características comportamentais de válvulas proporcionais e seus efeitos nos posicionadores eletro-hidráulicos, Master's thesis, Florianópolis, Brasil: Universidade Federal de Santa Catarina.

Pomar, M., Silveira, H. and Castelan, E. (2012). Condições lmis para análise e síntese de sistemas lineares sujeitos a uma classe de perturbações limitadas, Anais do XIX Congresso Brasileiro de Automática, pp. 2461-2466.

Tao, G. and Kokotović, P. (1994). Adaptive control of plants with unknown dead-zones, IEEE Transactions on Automatic Con-trol 39(1): 59-68. DOI: 10.1109/9.273339

Tarbouriech, S., Garcia, G., Gomes da Silva Jr, J. and Queinnec, I. (2011). Stability and stabilization of linear systems with saturating actuators, Springer-Verlag London.DOI: 10.1007/978-0-85729-941-3

Tarbouriech, S., Prieur, C. and da Gomes da Silva Jr., J. (2006). Stability analysis and stabilization of systems presenting nested saturations, IEEE Transaction Automatic Control 51(8): 1364-1371. DOI: 10.1109/TAC.2006.878743

Tarbouriech, S., Queinnec, I., Alamo, T., Fiacchini, M. and Camacho, E. (2011). Ultimate bounded stability and stabilization of linear systems interconnected with generalized saturated functions, Automatica 47(1): 1473-1481.DOI: 10.1016/j.automatica.2011.02.020

Tarbouriech, S., Queinnec, I. and Turner, M. (2009). Anti-windup design with rate and magnitude actuator and sensor saturations, European Control Conference, ECC09, pp. 330-335.

Turner, M. (2006). Actuator deadzone compensation: theoretical verification of an intuitive control strategy, Control Theory and Applications, IEE Proceedings 153(1): 59-68.

Valdiero, A., Guenther, R. and De Negri, V. (2005). New methodology for indentification of the dead zone in proportional directional hydraulic valves, Proceedings of the 18th International Congress of Mechanical Engeneering, pp. 377-384.

Vidyasagar, M. (1993). Nonlinear systems Theory, Prentice Hall International Editions.

Virvalo, T. (1997). Nonlinear model of analog valve, Proceedings of the 5th Scandinavian International Conference on Fluid Power, Vol. 3, pp. 199-213.

Young, P. and Willems, J. (1972). An approach to the linear multivariable servomechanism problem, International Journal of Control 15: 961-979. DOI: 10.1080/00207177208932211 